\title{
Comparative Evaluation of Implant Stability, Insertion Torque, and Implant Macrogeometry in Different Bone Densities Using Resonance Frequency Analysis
}

\author{
Syeda A Haseeb ${ }^{1}$, Kamala Rajendra², Litto Manual ${ }^{3}$, Anuraj S Kochhar ${ }^{4}$, Deepa Dubey ${ }^{5}$, Gagandeep S Dang ${ }^{6}$
}

\begin{abstract}
Aim and objective: Evaluation and comparison of insertion torque (IT) and the implant stability of two different implant macrogeometry in different bone densities using resonance frequency analysis.

Materials and methods: A total of 48 implants (with two implant types having regular and novel macrogeometry) were classified into 4 groups with 12 samples in each group. Group A regular implant without surface treatment, group B regular implant with surface treating, group C novel implant deprived of surface treating, and group $D$ was new dental implant with surface treatment were used. Implant stability quotient (ISQ), implant IT, removal torque (RT) percentage, and torque reduction percentage were calculated.

Results: The mean \pm SD ISQ value of bone 1 in group $A$ was $56.7 \pm 3.2$, in group $B$ was $58.6 \pm 2.4$, in group $C$ was $57.1 \pm 3.5$, and in group $D$ was $59.3 \pm 2.9$. In bone 2 , the value was $57.8 \pm 1.4,59.5 \pm 1.5,58.2 \pm 2.6$, and $59.5 \pm 2.4$ among $A, B, C$, and D groups correspondingly. In bone 3 , it was $59.4 \pm 2.4,60.3 \pm 2.3,60.4 \pm 2.8$, and $62.7 \pm 2.5$ among $A, B, C$, and D groups correspondingly. In bone 4, it was $67.2 \pm 3.4,69.5 \pm 2.7$, $68.7 \pm 2.4$, and $69.4 \pm 2.3$ among $A, B, C$, and D groups correspondingly. There was a substantial difference in IT and nonsignificant difference in $\mathrm{RT}$ in different groups.

Conclusion: There was a low IT value with new implant macrogeometry as compared to regular implant macrogeometry. There was absence of association between IT and implant stability.

Clinical significance: Calculation of torque insertion score helps in implant placement. Higher bone density scores produce a higher option of decreasing the initial torque. Low IT of new implant types is useful to reduce failure.

Keywords: Bone density, Implant, Surface treating, Torque.

The Journal of Contemporary Dental Practice (2021): 10.5005/jp-journals-10024-3118
\end{abstract}

\section{INTRODUCTION}

Dental implants are commonly used for the replacement of missing teeth. During the initial stage of healing after dental implant placement, it should be secured from unfavorable micromovements. As per the literature, this micromovement influences the osseointegration process. When micromovement exceeds threshold values of 50 and $150 \mu \mathrm{m}$, there is a higher chance of colonization of fibroblasts at the bone-implant interface, thus causing implant failure. In such conditions, the role of initial dental implant stability is important for a better outcome. These data of implant stability are useful in evaluating the time period required for complete healing around implant. This ensures treatment safety and decreases the overall treatment period. Various noninvasive techniques have been suggested to assess the implant stability, such as dental fine tester, periotest and implatest conventional impulse test, and resonance frequency analysis (RFA) and implant insertion torque (IT) measurements. ${ }^{1}$

Implant stability is used to measure the absence of clinical mobility of an implant, which is necessary for osseointegration. It can be attained by primary and secondary stabilities. The primary implant stability at the time of surgery is regulated by the surgical technique, bone density, and implant design. Secondary stability is based on bone development and remodeling at the implant-bone boundary and is prejudiced by the wound healing period. It has been stated that resonance frequency analysis (RFA) is a clinically valid and dependable technique to measure implant stability. ${ }^{2}$

\footnotetext{
${ }^{1}$ Department of Prosthodontics, Sharavathi Dental College and Hospital, Shivamogga, Karnataka, India

${ }^{2}$ Department of Dentistry, ESICMC PGIMSR Medical College, Bengaluru, Karnataka, India

${ }^{3}$ Department of Prosthodontics, Al-Azhar Dental College, Thodupuzha, Kerala, India

${ }^{4}$ Department of Orthodontics, Former Consultant, Max Hospital, Gurugram, Haryana, India

${ }^{5}$ Department of Oral Medicine and Radiology, Adesh Institute of Dental Sciences and Research, Bathinda, Punjab, India

${ }^{6}$ Dr Dang's Multispecialty Dental Clinic and Implant Centre, New Delhi, India

Corresponding Author: Kamala Rajendra, Department of Dentistry, ESICMC PGIMSR Medical College, Bengaluru, Karnataka, India, Phone: +919447612454, e-mail: drkamala81@gmail.com
}

How to cite this article: Haseeb SA, Rajendra $\mathrm{K}$, Manual L, et al. Comparative Evaluation of Implant Stability, Insertion Torque, and Macrogeometry in Different Bone Densities Using Resonance Frequency Analysis. J Contemp Dent Pract 2021;22(6):665-668.

Source of support: Nil

Conflict of interest: None

It is evident that implant-related factors, such as implant design, surface roughness, and patient-related factors, such as quantity and quality of bone, fit in patients' bone, and design of osteotomy, 
contribute to the success of the implant. Therefore, implant stability has to be adequate after insertion in the mouth for obtaining adequate osseointegration. ${ }^{3}$

Bone density determines the force required for dental implant insertion in bone. Other factors like size of the orifice and compressive stresses generated at the junction of dental implant and bone can affect the force. ${ }^{4}$ Thus the stability of the dental implant is greatly affected by compression stresses. It is mentioned in previous data that higher forces lead to bone ischemia and necrosis at the implant-tissue interface. Lower forces result in improved stability of the implant. Better osseointegration may result from selecting appropriate dental implants that comprise of correct implant size selection such as width and height. ${ }^{5}$

Continuous monitoring is the new concept of assessing implant stability which has replaced the old idea of microscopic or histologic analysis. ${ }^{6}$ In this study, we evaluated and compared the implant stability and torque insertion of two different implant macrogeometry in different bone densities quotient using resonance frequency analysis.

\section{Materials and Methods}

\section{Sample Selection}

The present experimental study was done in the department of Prosthodontics. In this in vitro study, we used $2 \times 2 \times 13 \mathrm{~cm}$ of four bone blocks of dense inflexible polyurethane foam of thickness 1 , 2 , and $3 \mathrm{~mm}$ and the fourth is completely cortical block. Since the $0.31 \mathrm{~g} / \mathrm{cm}^{3}$ is the average bone mineral density for the posterior maxilla and $0.55 \mathrm{~g} / \mathrm{cm}^{3}$ for the maxillary anterior area, hence $0.48 \mathrm{~g} / \mathrm{m}^{3}$ bone density polyurethane foam blocks were selected. Short fiber-filled epoxy sheets were involved as a cortical bone substitute. Two designs of the implant were as follows; the first one had trapezoidal threads having morse taper connection and a cervical portion with one millimeter of plane alignment (regular) and the second implant had similar characteristics plus healing chambers in the threads (new).

\section{Surface Treatment}

Surface treatment was given with a blasting process plus acid conditioning. Following this, blasting with titanium dioxide $\left(\mathrm{TiO}_{2}\right)$ microparticles having 45 to $110 \mu \mathrm{m}$ size was done. Final conditioning was performed with a solution of maleic acid proceeded by washing with water.

The surface roughness and morphology of four dental implants from each group were evaluated with atomic force microscopy and scanning electron microscopy. An optical laser profilometer was used for measuring surface roughness.

\section{Grouping of Implants}

We divided 48 implants into 4 groups (12 samples in each group) based on the design as well as surface treating. Group A had regular conical design which did not undergo surface treatment, group $B$ had normal conical plan which underwent surface treating, group $C$ was new conical design that did not undergo surface treating, and group D was novel conical plan which underwent surface treating.

\section{Experimental Design}

Implants were placed in homogeneous bone blocks with different bone densities following osteotomy preparation with a sequence of drilling was initially $2 \mathrm{~mm}$ drill, $3.5 \mathrm{~mm}$ conical drill, and $4.0 \mathrm{~mm}$ conical drill. A rise in the numerical size of the bone blocks denoted a raise in the bone density. Calculation of the implant stability quotient (ISQ) and IT scores was repetitive of four times for each bone density. In groups $A, B$, and $C$, the association between the implant stability and IT according to the size of the final drill diameter used was assessed. In group $D$, the association between the implant stability and IT conferring to the size of the final drill diameter $(4 \mathrm{~mm})$ in the bone blocks with an even cortical thickness of $1.5 \mathrm{~mm}$ was estimated. ${ }^{6}$

\section{Preparation, Osteotomy, and Fixture Installation}

All of the osteotomies were performed with a tender surgical method using a bench drill with a force of $20 \mathrm{~N}$ having $1000 \mathrm{rpm}$ speed with external abundant cooling. Following this, all implants were installed with a computed torquimeter machine.

\section{Measurements of IT and RT}

While connection, the peak IT was calculated for all tested implants, following the final seating of the fixtures using the Osstell Mentor (Osstell, Göteborg, Sweden). Each implant stability was measured in ISQ units. The RF values were symbolized in the ISQ in a scale from 1 to 100 and were averaged for each implant. Each measurement was performed up to four times and average of it was taken as final value. $^{6}$ The torque reduction (TR) percentage was calculated as TR $=$ insertion torque $(\mathrm{IT}) /$ removal torque $(\mathrm{RT})=100 \% / \mathrm{X}$.

\section{Statistical Analysis}

Data were tabulated and entered in MS excel sheet for inference using SPSS version (21.0). Results were interpreted as mean and standard deviations. $p$ value lower to 0.05 was deliberated substantial.

\section{Results}

Table 1 shows that group A had steady conical plane deprived of surface treating, group B had normal conical design with surface treating, group $C$ was novel conical plane deprived of surface treating, and group D was novel conical outline with surface treating.

Table 2 shows that mean \pm SD ISQ value of bone 1 in group $A$ was $56.7 \pm 3.2$, in group $B$ was $58.6 \pm 2.4$, in group $C$ was $57.1 \pm 3.5$, and in group $D$ was $59.3 \pm 2.9$. In bone 2 , the value was $57.8 \pm 1.4$, $59.5 \pm 1.5,58.2 \pm 2.6$, and $59.5 \pm 2.4$ among $A, B, C$, and $D$ groups correspondingly. In bone 3 , it was $59.4 \pm 2.4,60.3 \pm 2.3,60.4 \pm 2.8$ and $62.7 \pm 2.5$ among $A, B, C$, and D groups correspondingly. In bone 4, it was $67.2 \pm 3.4,69.5 \pm 2.7,68.7 \pm 2.4$, and $69.4 \pm 2$ among $A, B, C$, and D groups correspondingly.

Table 3 shows that mean \pm SD IT value of bone 1 was $13.2 \pm 2.2$, $13.6 \pm 2.3,1.4 \pm 2.5$, and $11.8 \pm 2.6$ among $A, B, C$, and $D$ groups correspondingly. In bone 2 , it was $16.1 \pm 1.5,16.3 \pm 1.6,13.2 \pm 2.3$, and $13.5 \pm 2.5$ among $A, B, C$, and $D$ groups correspondingly. In bone 3 , it was $19.2 \pm 2.6,20.3 \pm 2.8,15.2 \pm 3.2$, and $16.4 \pm 3.6$ among $A$, $B, C$, and D groups correspondingly. In bone 4 , it was $29.8 \pm 2.4$

Table 1: Distribution of implants in various groups

\begin{tabular}{lllll}
\hline Groups & Group $A$ & Group $B$ & Group C & Group D \\
\hline Design & $\begin{array}{l}\text { Regular conical } \\
\text { deprived of } \\
\text { surface treating }\end{array}$ & $\begin{array}{l}\text { Regular } \\
\text { conical with } \\
\text { surface } \\
\text { treating }\end{array}$ & $\begin{array}{l}\text { New } \\
\text { conical } \\
\text { deprived } \\
\text { of surface } \\
\text { treating }\end{array}$ & $\begin{array}{l}\text { New con- } \\
\text { ical with } \\
\text { surface } \\
\text { treating }\end{array}$ \\
Number & 12 & 12 & 12 & 12 \\
\hline
\end{tabular}


Table 2: Assessment of ISQ value in different groups

\begin{tabular}{lllll}
\hline Parameters & Bone 1 & Bone 2 & Bone 3 & Bone 4 \\
\hline Group A & $56.7 \pm 3.2$ & $57.8 \pm 1.4$ & $59.4 \pm 2.4$ & $67.2 \pm 3.4$ \\
Group B & $58.6 \pm 2.4$ & $59.5 \pm 1.5$ & $60.3 \pm 2.3$ & $69.5 \pm 2.7$ \\
Group C & $57.1 \pm 3.5$ & $58.2 \pm 2.6$ & $60.4 \pm 2.8$ & $68.7 \pm 2.4$ \\
Group D & $59.3 \pm 2.9$ & $59.5 \pm 2.4$ & $62.7 \pm 2.5$ & $69.4 \pm 2.3$ \\
$p$ value & 0.12 & 0.15 & 0.24 & 0.31 \\
\hline
\end{tabular}

$\mathrm{ISQ}$, implant stability quotient

Table 3: Assessment of insertion torque in different groups

\begin{tabular}{lllll}
\hline Parameters & Bone 1 & Bone 2 & Bone 3 & Bone 4 \\
\hline Group A & $13.2 \pm 2.2$ & $16.1 \pm 1.5$ & $19.2 \pm 2.6$ & $29.8 \pm 2.4$ \\
Group B & $13.6 \pm 2.3$ & $16.3 \pm 1.6$ & $20.3 \pm 2.8$ & $30.5 \pm 2.7$ \\
Group C & $11.4 \pm 2.5$ & $13.2 \pm 2.3$ & $15.2 \pm 3.2$ & $25.7 \pm 3.4$ \\
Group D & $11.8 \pm 2.6$ & $13.5 \pm 2.5$ & $16.4 \pm 3.6$ & $26.4 \pm 3.6$ \\
$p$ value & 0.05 & 0.02 & 0.01 & 0.01 \\
\hline
\end{tabular}

Table 4: Assessment of removal torque in different groups

\begin{tabular}{lllll}
\hline Parameters & Bone 1 & Bone 2 & Bone 3 & Bone 4 \\
\hline Group A & $6.4 \pm 1.3$ & $7.2 \pm 1.2$ & $8.4 \pm 1.2$ & $20.3 \pm 1.3$ \\
Group B & $6.9 \pm 1.7$ & $7.4 \pm 1.4$ & $9.7 \pm 1.3$ & $20.5 \pm 1.4$ \\
Group C & $6.6 \pm 1.4$ & $7.3 \pm 1.6$ & $8.2 \pm 1.4$ & $18.3 \pm 1.4$ \\
Group D & $6.7 \pm 1.2$ & $7.5 \pm 1.7$ & $8.4 \pm 1.6$ & $19.3 \pm 1.2$ \\
$p$ value & 0.91 & 0.82 & 0.72 & 0.65 \\
\hline
\end{tabular}

$30.5 \pm 2.7,25.7 \pm 3.4$, and $26.4 \pm 3.6$ among $A, B, C$, and D groups correspondingly.

Table 4 shows that mean \pm SD RT value of bone 1 was $6.4 \pm 1.3$, $6.9 \pm 1.7,6.6 \pm 1.4$, and 6.7 c 1.2 among $A, B, C$, and D groups correspondingly. In bone 2 was $7.2 \pm 1.2,7.4 \pm 1.4,7.3 \pm 1.6$, and $7.5 \pm 1.7$ among $A, B, C$, and $D$ groups correspondingly. In bone 3 , it was $8.4 \pm 1.2,9.7 \pm 1.3,8.2 \pm 1.4$, and $8.4 \pm 1.6$ among $A, B$, $C$, and D groups correspondingly. In bone 4, it was $20.3 \pm 1.23$, $20.5 \pm 1.4,18.3 \pm 1.4$, and $19.3 \pm 1.2$ among $A, B, C$, and $D$ groups correspondingly. The variance was nonsubstantial $(p>0.05)$. It is found that RT values were $45.2 \%$ smaller than the torque insertion for the groups with normal macrogeometry (group A and group B) and $38.6 \%$ for the groups with the novel implant macrogeometry (group C and group D).

Figure 1 shows that there was significant difference $(p<0.05)$ in mean torque insertion values of two implant macrogeometries (regular, new) in different bone types. Table 5 shows that TR percentage in groups $A$ and $B$ found to be $52 \%$ and in groups $C$ and $D$ was $40 \%$ in bone type 1 . In bone type 2 , it was $54 \%$ and 46 among $A, B, C$, and $D$ groups correspondingly. In type 3 bone, it was $53 \%$ and $48 \%$ among $A, B, C$, and D groups correspondingly. In type 4 bone, it was $33 \%$ in all groups.

Table 6 shows that there was no correlation existed between torque insertion and stability (ISQ) values in all groups.

\section{Discussion}

Surface treatment of dental implants significantly enhances the chances of success. Titanium dioxide is widely used for this purpose. ${ }^{7}$ We evaluated and compared the IT and the implant stability of two different implant macrogeometry, i.e., regular

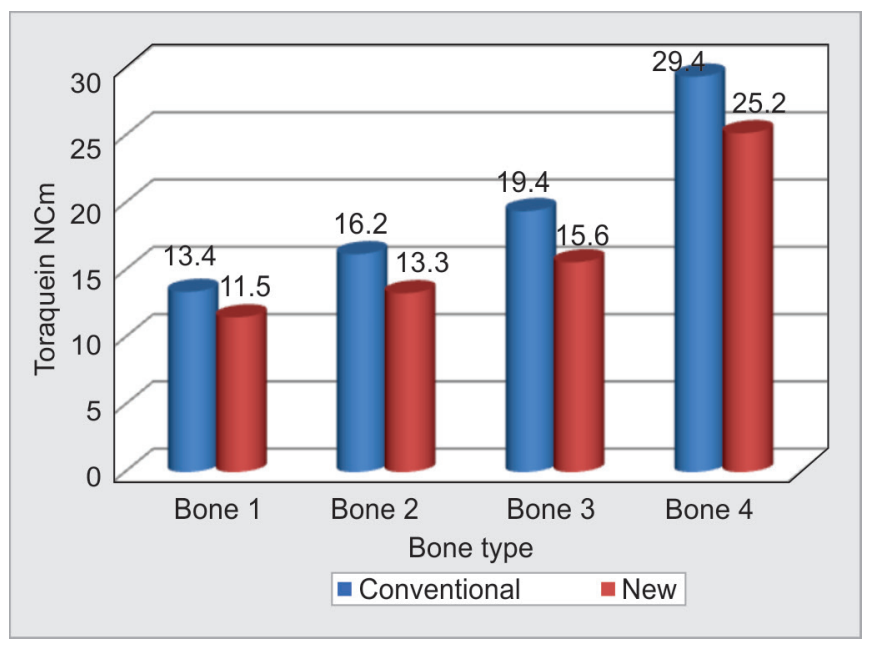

Fig. 1: Insertion torque values in two implant macrogeometries

Table 5: Torque reduction percentage

\begin{tabular}{lll}
\hline Bone type & Group A and Group B & Group C and Group D \\
\hline 1 & 52 & 40 \\
2 & 54 & 46 \\
3 & 53 & 48 \\
4 & 33 & 33 \\
\hline
\end{tabular}

Table 6: Pearson correlation analysis in all bone type in different groups

\begin{tabular}{lllll}
\hline Groups & Bone 1 & Bone 2 & Bone 3 & Bone 4 \\
\hline Group A & $R=0.32$, & $R=-0.23$, & $R=-0.31$, & $R=-0.47$, \\
& $p-0.41$ & $p-0.42$, & $p-0.41$ & $p-0.01$ \\
Group B & $R=0.36$, & $R=0.09$, & $R=-0.72$, & $R=-0.16$, \\
& $p-0.42$ & $p-0.92$ & $p-0.42$ & $p-0.82$ \\
Group C & $R=0.04$, & $R=-0.54$, & $R=-0.43$, & $R=-0.43$, \\
& $p-0.12$ & $p-0.04$ & $p-0.12$ & $p-0.42$ \\
Group D & $R=0.07$, & $R=0.42$, & $R=-0.05$, & $R=-0.32$, \\
& $p-0.81$ & $p-0.32$ & $p-0.81$ & $p-0.45$ \\
\hline
\end{tabular}

and new in different bone densities quotient using resonance frequency analysis.

Jimbo et al. ${ }^{8}$ suggested that new macrogeometry increases the osseointegration. We used new macrogeometry implants having healing chambers. This design helped in decompression of bone by creating spaces. This is helpful in obtaining better implant stability.

Gehrke et al. assessed the RT, IT, and the stability of implant using RFA in dissimilar polyurethane block masses of two implant macrogeometries. Authors found nonsignificant differences $(p>0.05)$ in ISQ values in all bone models. It was $57.7 \pm 3.0$ in bone type 1,58.6 \pm 2.2 in bone $2,60.6 \pm 2.3$ in bone 3 , and $68.5 \pm 2.8$ in bone 4 . Regular macrogeometry implant exhibited higher values of IT compared to new implant macrogeometry without disturbing the values of implant stability. They concluded that primary stability depends on both bone quality and IT. ${ }^{9}$ This is in accordance to our findings.

Implant stability may be decided by means of RFA method with Osstell Mentor. This is simple and precise chair side portable, hand-held diagnostic device, commonly used in clinical practice to assess impeding failure and to measure implant stability. Using 
this method data can be recorded. It works on the magnetic frequencies. RFA is a clinical technique which can quantitatively evaluate primary implant stability so as to recognize implants with little stability and to take precautions to develop it to choose on healing method (one stage or two stages), to adopt for suitability of instant/early loading, and to decide healing time before loading. ${ }^{2}$ Sennerby et al. stated in their review article that the RFA method can be used at any stage of implant treatment as supplementary parameter to upkeep conclusion-making. ${ }^{10}$ Gehrke et al. found RFA as an effective noninvasive method to evaluate implant stability. ${ }^{9}$ Similarly Kanth et al., ${ }^{2}$ Satwalekar et al., ${ }^{11}$ and Bafijari et al. ${ }^{12}$ stated that RFA is non-invasive, reliable clinically, user-friendly method which has repeatability, reproducibility, and reliable for identifying risk of implant failure and implant stability during early healing.

We found that significant difference in IT value in all bone types for all groups. Acil et al. ${ }^{13}$ found that macrogeometry, such as design and topography, affects the interaction between dental implants and bone. It is also mentioned that surface treatment of dental implants can determine the implant stability.

We found that RT value was comparatively higher in bones 3 and 4 as compared to bones 1 and 2 in all groups. We observed that RT values were $45.2 \%$ smaller than the torque insertion for the groups with normal macrogeometry (groups $A$ and $B$ ). It is due to the fact that there is decrease in stress on bone because of availability of decompression chambers. As reported by Ahn et al., ${ }^{14}$ limited viscoelastic possessions of the neighboring artificial bone outcomes in the variance between torque insertion and torque removal. This leads to less resistance during removal. Less viscosity was seen in bone 4 whereas more viscous was seen in bone 3 having greater TR.

In our study, it was evident that TR percentage was $52 \%$ in groups $A$ and $B$ and $40 \%$ in groups $C$ and $D$ in type 1 bone. In type 2 bone, it was $54 \%$ and $46 \%$ among $A, B, C$, and $D$ groups correspondingly. In contrast to our results, Gedrange et al. suggested that various surface treatments of dental implants may not always be detected by RFA and this technique has low sensitivity. The value of resonance frequency is affected by both the level of the bone surrounding the fixture and the stiffness of the interface between implant and bone. ${ }^{15}$

In present study, we did not find correlation between torque insertion and stability (ISQ) scores in all groups. Our results are in association with various studies which mentioned that the higher bone density scores produce a higher option of decreasing the initial torque calculated by the viscosity of tissue. ${ }^{16,17}$

The limitation of the study is small sample size and it has not compared the other noninvasive technique to check implant stability and the study was in vitro evaluation. Further clinical studies are in need for to evaluate the accuracy of RFA, torque value, and implant stability.

\section{Conclusion}

Low IT of new implant types is useful to reduce failure as compared to regular implant macrogeometry. There was no association between insertion torque and stability of implant. With respect to clinical relevance, calculation of insertion torque score helps in implant placement and to reduces the risk of implant failure. Higher bone density scores produce a higher option of decreasing the initial torque. Future prospects should be directed for resonance frequency analysis since it is reliable, simple device used for assessment of dental implant stability. Large scale studies are required to substantiate further utility.

\section{References}

1. Baldi D, Lombardi T, Colombo J, et al. Correlation between insertion torque and implant stability quotient in tapered implants with knife-edge thread design. BioMed Res Int 2018;2018:7201093. DOI: 10.1155/2018/7201093.

2. Kanth KL, Swamy DN, Mohan TK, et al. Determination of implant stability by resonance frequency analysis device during early healing period. J NTR Univ Health Sci 2014;3:169-175. DOI: 10.4103/22778632.140936.

3. Stacchi C, Vercellotti T, Torelli L, et al. Changes in implant stability using different site preparation techniques: twist drills versus piezosurgery: a single-blinded, randomized, controlled clinical trial. Clin Implant Dent Relat Res 2013;15:188-197. DOI: 10.1111/j.1708-8208.2011.00341.x.

4. Nedir R, Bischof M, Szmukler-Moncler S, et al. Predicting osseointegration by means of implant primary stability. Clin Oral Implants Res 2004;15:520-528. DOI: 10.1111/j.1600-0501.2004.01059.x.

5. Javed F, Romanos GE. The role of primary stability for successful immediate loading of dental implants. A literature review. J Dent 2010;38:612-620. DOI: 10.1016/j.jdent.2010.05.013.

6. Bayarchimeg $D$, Namgoong $H$, Kim BK, et al. Evaluation of the correlation between insertion torque and primary stability of dental implants using a block bone test. J Periodontal Implant Sci 2013;43:30-36. DOI: 10.5051/jpis.2013.43.1.30.

7. Devlin H, Horner K, Ledgerton DA. Comparison of maxillary and mandibular bone mineral densities. J Prosthet Dent 1998;79:323-327. DOI: 10.1016/s0022-3913(98)70245-8.

8. Jimbo R, Tovar N, Anchieta RB, et al. The combined effects of undersized drilling and implant macrogeometry on bone healing around dental implants: an experimental study. Int J Oral Maxillofac Surg 2014;43:1269-1275. DOI: 10.1016/j.ijom.2014.03.017.

9. Gehrke SA, Pérez-Díaz L, Mazón P, et al. Biomechanical effects of a new macrogeometry design of dental implants: an in vitro experimental analysis. J Funct Biomater 2019;10(4):47. DOI: 10.3390/jfb10040047.

10. Sennerby L. Resonance frequency analysis for implant stability measurements. A review. Integr Diagn Update 2015;1:1-11. DOI: 10.4103/jdi.jdi_7_18.

11. Satwalekar P, Nalla S, Reddy R, et al. Clinical evaluation of osseointegration using resonance frequency analysis. Indian Prosthodont Soc 2015;15:192-199. DOI: 10.4103/0972-4052.165171.

12. Bafijari D, Benedetti A, Stamatoski A, et al. Influence of resonance frequency analysis (RFA) measurements for successful osseointegration of dental implants during the healing period and its impact on implant assessed by Osstell mentor device. Open Access Maced J Med Sci 2019;7(23):4110-4115. DOI: 10.3889/oamjms.2019.716.

13. Acil Y, Sievers, J, Gülses A, et al. Correlation between resonance frequency, insertion torque and bone-implant contact in selfcutting threaded implants. Odontology 2017;105:347-353. DOI: 10.1007/s10266-016-0265-2.

14. Ahn SJ, Leesungbok R, Lee SW, et al. Differences in implant stability associated with various methods of preparation of the implant bed: an in vitro study. J Prosthet Dent 2012;107:366-372. DOI: 10.1016/S0022-3913(12)60092-4.

15. Gedrange T, Hietschold V, Mai R, et al. An evaluation of resonance frequency analysis for the determination of the primary stability of orthodontic palatal implants. A study in human cadavers. Clin Oral Implants Res 2005;16:425-431. DOI: 10.1111/j.1600-0501.2005.01134.x.

16. Sennerby L, Meredith N. Implant stability measurements using resonance frequency analysis: biological and biomechanical aspects and clinical implications. Periodontology 2008;47:51-66. DOI: 10.1111/j.1600-0757.2008.00267.x.

17. Rabel A, Köhler SG, Schmidt-Westhausen AM. Clinical study on the primary stability of two dental implant systems with resonance frequency analysis. Clin Oral Investig 2007;11:257-265. DOI: 10.1007/s00784-007-0115-2. 